\title{
Why does low-luminosity AGN fueling remain an unsolved problem?
}

\author{
Paul Martini ${ }^{1}$ \\ ${ }^{1}$ Harvard-Smithsonian Center for Astrophysics; 60 Garden Street, MS20; \\ Cambridge, MA 02138, USA email:pmartini@cfa.harvard.edu
}

\begin{abstract}
Despite many years of effort, observational studies have not found a strong correlation between the presence of any proposed fueling mechanism and low-luminosity AGN. After a discussion of the mass requirements for fueling, I summarize this observational work and provide a number of hypotheses for why the nature of AGN fueling has remained unresolved. In particular, I stress the potential importance of the increasing number of candidate fueling mechanisms with decreasing mass accretion rate, the relevant spatial scales for different fueling mechanisms, and the lifetime of an individual episode of nuclear accretion. The episodic AGN lifetime is a particularly relevant complication if it is comparable to or shorter than the time that the responsible fueling mechanisms are observationally detectable. I conclude with a number of relatively accessible areas for future investigation.
\end{abstract}

\section{Introduction}

One of the main, unsolved problems in AGN research is how the AGN fuel is transported to the central, supermassive black hole. For low-luminosity AGN, the likely source of this fuel is the host galaxy itself, and in particular the ISM. As most of this material is distributed in a rotating disk extending out to kiloparsec scales, the problem of AGN fueling is essentially a problem of angular momentum transport: All but approximately one part in $10^{7}$ of the angular momentum must be removed for material to flow from kiloparsec scales to the event horizon on AU scales.

The problem has remained unsolved in part because the most relevant spatial scales for fueling, the central parsec and inward, are only observable in the very nearest galaxies. An angular resolution of $0.1^{\prime \prime}$ only corresponds to $1 \mathrm{pc}$ or better spatial resolution out to a distance of $2 \mathrm{Mpc}$. Even the central $10 \mathrm{pc}$ are not readily resolved for large samples of nearby AGN. The present technical limitations on angular resolution have therefore made it impossible to study AGN fueling directly and instead driven investigations to indirect, statistical studies of active and inactive galaxy samples in order to identify the mechanism(s) responsible for fueling accretion.

\subsection{Requirements for Fueling}

Before consideration of these investigations, it is valuable to first consider the mass accretion rates estimated to produce the observed population of low-luminosity AGN, which are defined here to be any AGN less luminous than a QSO. The luminosity of an AGN is related to the mass accretion rate by $L=\epsilon \dot{M} c^{2}$, where $\epsilon$ is the radiative efficiency of the accretion. This accretion is commonly assumed to occur via a Shakura-Sunyaev thin disk (Shakura \& Sunyaev 1973) with a constant radiative efficiency $\epsilon=0.1$. To maintain Eddington accretion onto a supermassive black hole requires a mass accretion rate of

$$
\dot{M}_{E d d}=0.2 \epsilon_{0.1}^{-1}\left(\frac{M}{10^{7} \mathrm{M}_{\odot}}\right) \mathrm{M}_{\odot} \mathrm{yr}^{-1}
$$


where $\epsilon_{0.1}=\epsilon / 0.1$. The mass accretion rate is commonly parametrized in terms of the Eddington rate as the dimensionless accretion rate $\dot{m} \equiv \dot{M} / \dot{M}_{E d d}$, similarly the bolometric luminosity can be expressed as $l \equiv L_{b o l} / L_{E d d}$. For a standard accretion disk $l=\dot{m}$, while lower efficiency ADAF models, which likely become important below $\dot{m}=\dot{m}_{\text {crit }} \sim 0.01$, predict $l \propto \dot{m}^{2}$ from a scaling of $\epsilon_{0.1}=\dot{m} / \dot{m}_{\text {crit }}$ (Narayan, Mahadevan, \& Quataert 1998). The required mass accretion rates from fueling may be even higher if ADIOS/CDAF models are important, as these models predict that only a small fraction of the supplied mass is actually accreted by the black hole (Blandford \& Begelman 1999). The key importance of these relations is that the mass accretion rates required for low-luminosity AGN do not decline as rapidly as their luminosity.

The most luminous low-luminosity AGN, Seyfert 1s, appear to have central, supermassive black holes with $M_{\bullet}=10^{7} \mathrm{M}_{\odot}$ (Ferrarese et al. 2001) and $l=0.1$, which corresponds to accretion at $\dot{m}=0.1$ or $\dot{M}=0.02 \mathrm{M}_{\odot} / \mathrm{yr}$. More typical Seyferts, which constitute a total of approximately $10 \%$ of the luminous galaxy population (Ho, Filippenko, \& Sargent 1997 ) likely accrete with $\dot{m}=0.01$, while the $30 \%$ of the luminous galaxy population that are LINERs likely accrete with $\dot{m}=10^{-2}-10^{-4}$, based on estimates of $l$ (Ho 1999). If all luminous galaxies go through periodic episodes as AGN, then over a time period of $10^{8}$ years $\dagger$, a $10^{7} \mathrm{M}_{\odot}$ black hole will appear as a Seyfert galaxy for a total of $10^{7} \mathrm{yr}$ and accrete on order $10^{3} \mathrm{M}_{\odot}$, appear to be a LINER for $3 \times 10^{7}$ yr and accrete $10^{3} \mathrm{M}_{\odot}$, and appear to be inactive for $6 \times 10^{7} \mathrm{yr}$. Therefore the mass inflow rates and total mass reservoirs required to power low-luminosity AGN are relatively meager.

\subsection{Proposed Fueling Mechanisms}

Figure 1 lists the many mechanisms proposed to drive angular momentum transport in the host galaxy and provide fuel to the central parsec. These mechanisms can be divided between gravitational and hydrodynamic mechanisms. Gravitational mechanisms, such as galaxy interactions (Toomre \& Toomre 1972) and large-scale bars (Simkin, Su, \& Schwarz 1980), remove angular momentum through torques, while hydrodynamic mechanisms, such as turbulence in the ISM (Elmegreen et al. 1998), remove angular momentum through gas dynamical effects. Many of these mechanisms are discussed in the review by Shlosman, Begelman, \& Frank (1990) and the more recent review by Wada (2004). The latter in particular provides an excellent overview of recent theoretical work on hydrodynamic fueling mechanisms and places particular emphasis on high resolution simulations of the multiphase ISM in the central kiloparsec.

While a large number of mechanisms have been proposed for AGN fueling, the vast majority are only likely to provide relatively low mass accretion rates. Mergers between galaxies, particularly major mergers, is the mechanism most commonly invoked to explain the high accretion rates required to power luminous QSOs, while at the somewhat lower mass accretion rates responsible for Seyfert-level luminosities, mechanisms such as bars and minor mergers have also been considered. A progressively larger number of candidate mechanisms could be important at yet lower mass accretion rates. The mechanisms listed in Figure 1 are approximately ordered by their relative maximum mass accretion rates. As the mass accretion rate to the nucleus is expected to gradually taper off, rather than stop abruptly, mechanisms invoked to explain high mass accretion rates may also produce low mass accretion rates as the fuel supply is gradually depleted.

$\dagger$ This time period is only adopted to illustrate the potential total fuel requirements of a low-luminosity AGN. The relative numbers of Seyferts and LINERs only constrain the duty cycle of these phases and not the lifetime 


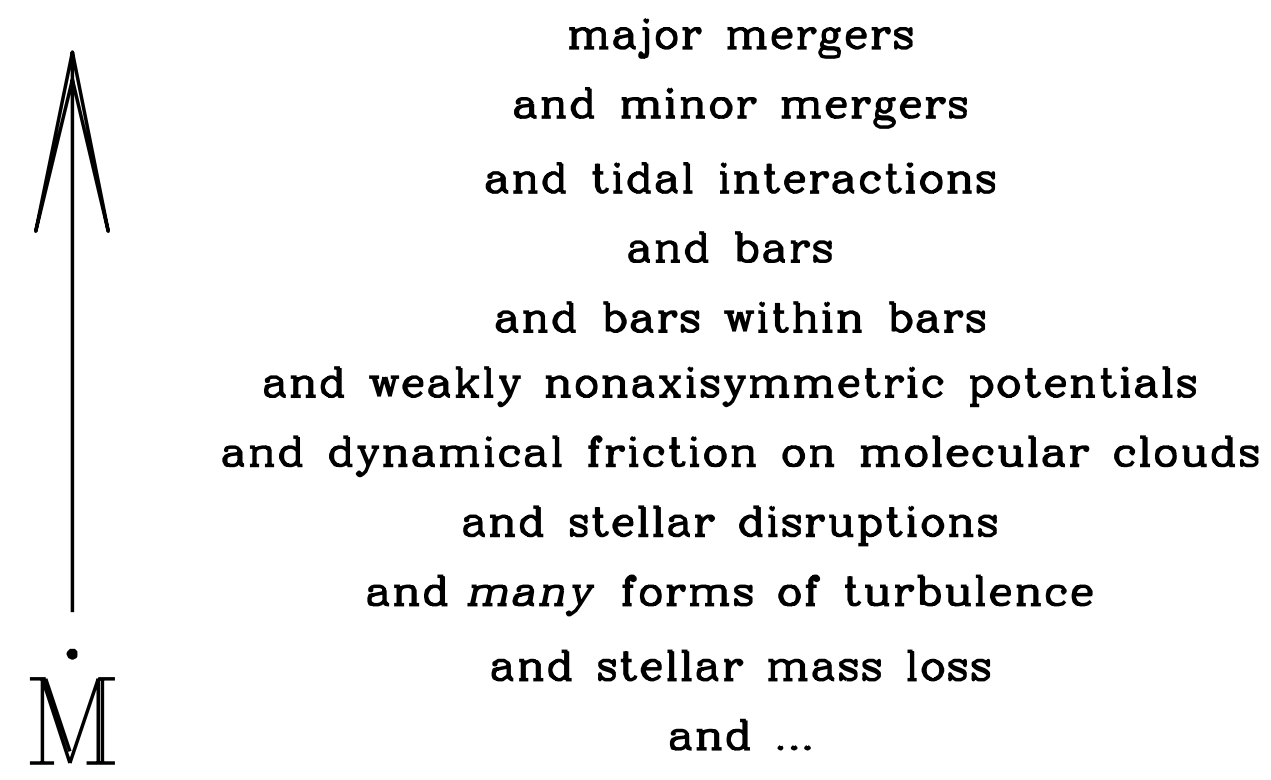

Figure 1. Mechanisms proposed to fuel accretion onto black holes at the centers of galaxies. The fueling mechanisms are approximately ordered by their expected maximum accretion rate. Progressively larger numbers of mechanisms of progressively more common mechanisms may be responsible for supplying the lowest rates of accretion. This suggests that while the relevant question for luminous AGN may be "Why are active galaxies active?" the question should be "Why are inactive galaxies inactive?" at the lowest accretion rates.

\subsection{Observational Searches}

As the spatial scales most important for fueling are currently inaccessible in most galaxies, searches have instead employed large samples of AGN and inactive, control galaxies, to identify the fueling mechanism(s). Ideally, a candidate fueling mechanism should only be found in the AGN sample. These experiments are challenging in that they require both large samples of galaxies and very careful control of systematic effects in the selection of both the AGN and the control samples. Claims over the years of statistically significant excesses have all diminished after reevaluation of sample sizes and the details of the AGN and control sample selection (e.g. different distance distributions, host galaxy types). Sample selection remains a very important consideration as progressively more AGN are found in progressively more sensitive surveys (e.g. Ho et al. 1997) and few studies of AGN fueling use samples selected with uniform and unbiased criteria, such as hard X-ray luminosity, nor do they have comparably sensitive observations of the inactive, control sample.

The two main and most readily observed 'large-scale' mechanisms are bars and interactions. Neither of these features is seen in significant excess in AGN samples compared to carefully-matched control samples (Fuentes-Williams \& Stocke 1988; Mulchaey \& Regan 1997; Schmitt 2001; see also Schmitt, these proceedings). One explanation for these results is that AGN fueling is predominantly mitigated by smaller-scale phenomenon than accessible in these ground-based surveys (e.g. Martini \& Pogge 1999), a point illustrated in part by Figure 1. While the fueling mechanisms are approximately ordered by accretion 
rate, the mechanisms proposed to produce lower accretion rates are also progressively smaller-scale phenomena and more difficult to identify observationally. The desire for finer spatial resolution motivated a careful study of the circumnuclear region (on $100 \mathrm{pc}$ scales) with HST, but still no significant differences between AGN and control samples were found (Martini et al. 2003a).

\section{Why have surveys been unsuccessful?}

The null results from searches for AGN fueling mechanism(s) have successfully eliminated many simple models for AGN fueling, such as the importance of a single mechanism and the hypothesis that the AGN lifetime is comparable to the Hubble time. Several relatively straightforward scenarios that can explain the present null results include the importance of multiple fueling mechanisms, the overly broad classifications for fueling mechanisms, correlations between fueling mechanism and fueling rate, and the importance of time dependence. Here I discuss each of these scenarios in turn and describe both how they can explain current observations and be tested by future work.

1. Multiple fueling mechanisms are important: This scenario is the simplest explanation of current observational results. If more than one mechanism is important (e.g. bars and interactions), then the significance of the correlation between any one mechanism and AGN will be diluted by AGN fueled by other mechanisms. There is good reason to consider this possibility, as the theoretical motivations for multiple mechanisms are equally plausible. This scenario could be tested with the 'classical' survey approach.

2. Current classifications for fueling mechanisms are too broad: Observational work has already shown that there is a wide dispersion in the detailed properties of mechanisms proposed for fueling, such as the fact that not all barred galaxies possess offset shocks that connect to grand-design nuclear spiral structure (Martini et al. 2003b). If only certain subclasses of a candidate fueling mechanism actually remove angular momentum (e.g. only certain types of bars), then the correlation between this broader class and AGN will be diluted. This rationale could also explain the absence of AGN in some barred and/or interacting galaxies. Reevaluation of surveys for the incidence of certain types of bars, for example only strong bars, is a simple test of this scenario.

3. There are correlations between fueling mechanism and fueling rate: As noted above in the discussion of Figure 1, the number of proposed fueling mechanisms increases as the required accretion rates diminish. Present surveys do not explicitly consider low-luminosity AGN with a specific range of $\dot{M}$ or $\dot{m}$. For example, Figure 1 suggests that it might be easier to identify the fueling mechanism for higher accretion rates, both because the relevant mechanisms may be fewer in number and they are visible on larger scales. Thus inclusion of all low-luminosity AGN would dilute any potential signal for the high accretion rate subsample. The importance of correlations between fueling mechanisms and fueling rates could be tested by specifically identifying samples of higher accretion rate AGN with empirical techniques to estimate $M_{\bullet}$ combined with multiwavelength observations to estimate $L_{b o l}$. The main difficulty of this approach is that more luminous, higher accretion rate objects are rarer and on average further away. The observed spatial resolution is thus poorer, in addition to the greater contamination of the circumnuclear region by the brighter nuclear point source.

Lower accretion rate objects are progressively more challenging to study due to the larger pool of candidate mechanisms, the difficulty in quantitatively classifying many of the proposed fueling mechanisms (e.g. nonaxisymmetric potentials, dynamical friction on molecular clouds), and the small spatial scales relevant for most of the mechanisms. Lower mass accretion rates in particular are likely to be controlled by small-scale, essentially 
stochastic processes such as the distribution of molecular clouds and the time since the last supernova. For these largely hydrodynamic processes, Wada (2004) finds the accretion rate into the nuclear region $(<1 \mathrm{pc})$ varies over three orders of magnitude on a fluctuation timescale of $10^{4}-10^{5} \mathrm{yr}$, due to the inhomogeneity of the circumnuclear region. At the lowest mass accretion rates, no correlation between various fueling mechanisms and nuclear activity may be found unless the central parsec can be resolved.

4. Time dependence is important: The above three methods illustrate minor complications that can plausibly explain why existing surveys to identify the AGN fueling mechanism have been unsuccessful. These complications can in principle be tested with more careful sample selection and classification of the various mechanisms proposed for AGN fueling. However, an alternate, physically plausible explanation for the null results to date is that time dependence is important. If the AGN lifetime is comparable to or shorter than the inflow time due to a given fueling mechanism, studies of the frequency of some fueling mechanism(s) in active and inactive galaxies will show no correlation with activity, even if the correct fueling mechanism has been identified.

The potential importance of time-dependence is illustrated in Figure 2 with a simple cartoon of azimuthally-averaged inflow rate as a function of radius. Here I have assumed that several different mechanisms are driving inflow at a range of different galactic radii. At time $t_{1}$, mass loss from stars provides fuel to produce the spectral signatures of a LINER, while at larger scales higher inflow rates are produced by gravitational torques. By some later time $t_{2}$, accretion in the nuclear region has switched off and no AGN is visible, yet the gravitational torques continue to drive inflow toward the galactic nucleus. At this time, the galaxy would be classified as barred, yet not as an AGN. Eventually, at time $t_{3}$ the greater level of inflow from the gravitational torques reaches the central black hole and produces a sufficiently luminous AGN for the galaxy to be classified as a Seyfert. Without providing actual units for time, mass accretion rate, or radius, the progression from $t_{1}-t_{3}$ in this example illustrates how the classical approach to the identification of the AGN fueling mechanism(s) could be completely thwarted if the episodic lifetime of an AGN is comparable to the lifetime of one or more important fueling mechanisms. The final panel is intended to illustrate the likely complexity of the actual phenomenon, rather than an actual physical scenario.

\section{Future Directions}

The observational studies discussed here have eliminated many simple models for AGN fueling and led to a much clearer appreciation of the complexity of the problem. Current and future observational facilities, new empirical methods to estimate black hole masses, as well as computational improvements, enable several new lines of investigation.

Detailed studies of the circumnuclear region: The observational studies described above have revealed significant correlations between the circumnuclear dust morphology and the presence or absence of a large-scale bar. Further work to understand the formation of nuclear spiral structure would be of great value, particularly measurements of the pitch angle and mass surface density with radius, as well as the distribution of molecular clouds. Kinematic data for many disk galaxies with higher spatial resolution is also needed, both to measure rotation curves and hence the mass distribution at small physical scales for accurate theoretical models, and also to quantify the extent to which the gas kinematics in the centers of galaxies are chaotic. While the best resolution will be obtained with future ALMA observations, significant progress will be possible in the near future with CARMA and the SMA, as well as with the completion of current surveys of nearby galaxies such as NUGA (Combes et al. 2004). 

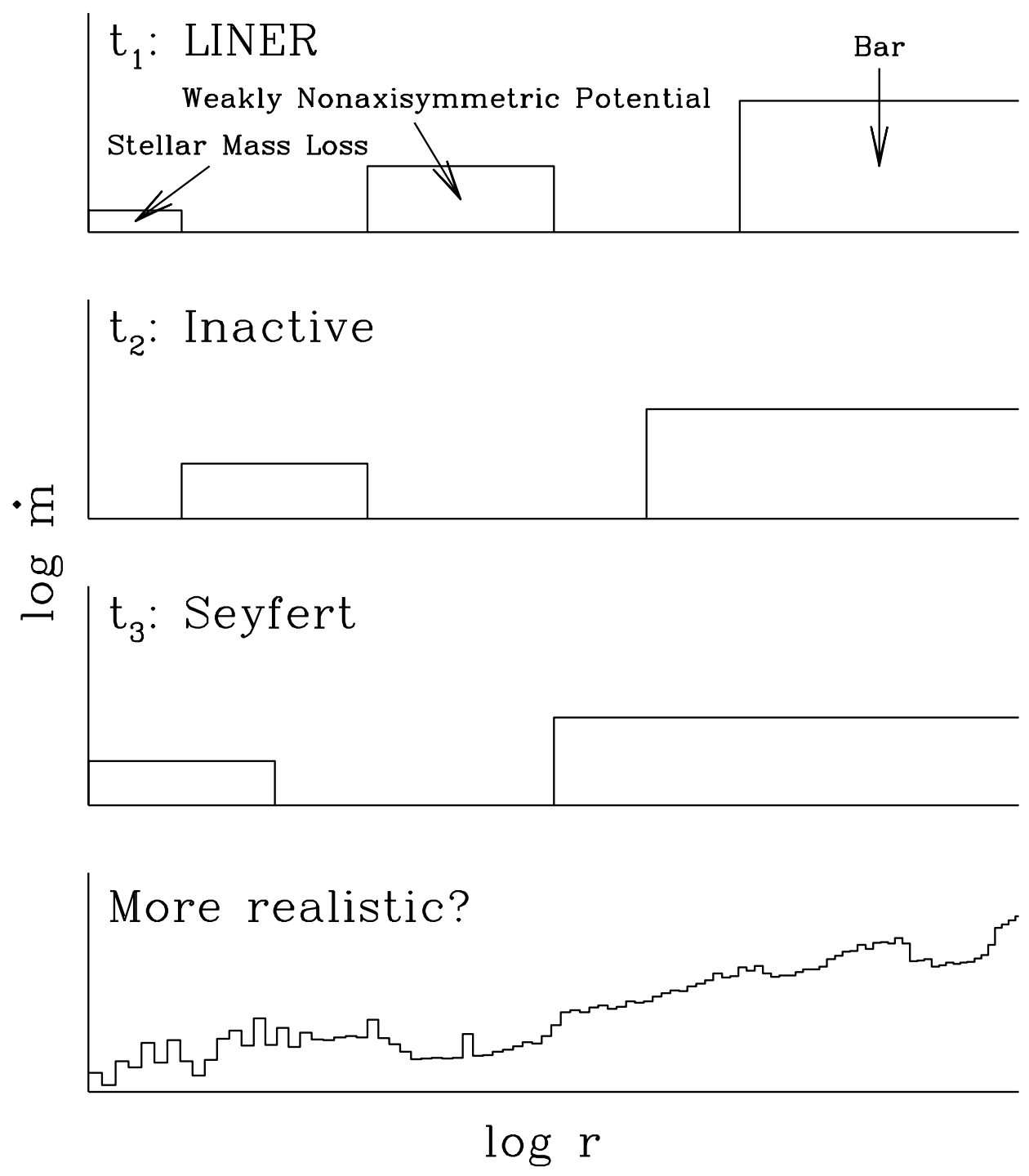

Figure 2. Simple cartoon illustrating the potential importance of time evolution in AGN fueling. The top three panels present snapshots of the mass accretion rate as a function of radius at three separate times, and illustrate how the presence of fueling mechanisms on larger spatial scales may be decoupled from the presence of an AGN if the episodic AGN timescale is comparable to or shorter than the inflow timescale due to the relevant fueling mechanism. The bottom panel illustrates the likely complexity of inflow rate as a function of radius for an actual galaxy.

Physically defined samples: The existence of empirical techniques to estimate black hole masses make it possible to reconsider AGN fueling with samples defined by $M_{\bullet}$ and $L_{b o l} / L_{E d d}$. In particular, success in identifying strong evidence for any AGN fueling mechanism may first come from investigation of samples with higher mass accretion rates. This work, and all surveys that employ the classical approach of AGN and inactive, control samples, also require extremely careful selection of the samples. In particular, it 
is extremely important to have an unbiased and uniform selection of both the AGN and control sample, for example based on hard X-ray luminosity.

Improved hydrodynamic simulations of the circumnuclear regions: Current observations can probe AGN with a spatial resolution as fine as tens of parsecs. This spatial resolution is comparable or only slightly worse than that achieved by current multiphase ISM models on galaxy scales (e.g. Wada, these proceedings). Increased spatial resolution in the simulations, along with estimates of the mass accretion rates at different spatial scales and any correlations with observable fueling mechanisms, would be very useful for interpreting the present observations. Reconsideration of mass inflow in bars and mergers in models with high spatial resolution and self-consistent star formation would also be of great value, as would velocity field calculations for the interpretation of two-dimensional kinematic observations, and further consideration of the time domain.

While surveys to identify the mechanisms responsible for AGN fueling have provided a string of null results, the lessons learned from these investigations provide several clear and observationally testable hypotheses. New work based on large samples with empirical estimates of black hole masses, multiwavelength measurements of $L_{b o l}$, unbiased selection criteria, and careful definition of candidate fueling mechanisms offers the promise of significant progress toward solving the problem of how low-luminosity AGN are fueled.

\section{Acknowledgements}

I acknowledge support from HST grant AR-9547, the International Astronomical Union, and the conference organizers. I would also like to thank Rick Pogge for helpful comments and discussions.

\section{References}

Blandford, R. D., \& Begelman, M. C. 1999, MNRAS, 303, L1

Combes, F., et al. 2004, A\&A, 414, 857

Elmegreen, B. G., et al. 1998, ApJ, 503, L119

Ferrarese, L., Pogge, R. W., Peterson, B. M., Merritt, D., Wandel, A., \& Joseph, C. L. 2001, ApJ, 555, L79

Fuentes-Williams, T., \& Stocke, J. 1988, AJ, 96, 1235

Ho, L. C., Filippenko, A. V., \& Sargent, W. L. 1997, ApJS, 112, 315

Ho, L. C. 1999, ApJ, 516, 672

Martini, P., \& Pogge, R. W. 1999, AJ, 118, 2646

Martini, P., Regan, M. W., Mulchaey, J. S., \& Pogge, R. W. 2003a, ApJ, 589, 774

Martini, P., Regan, M. W., Mulchaey, J. S., \& Pogge, R. W. 2003b, ApJS, 146, 353

Mulchaey, J. S., \& Regan, M. W. 1997, ApJ, 482, L135

Narayan, R., Mahadevan, R., \& Quataert, E. 1998 in The Theory of Black Hole Accretion Discs, (eds. M. A. Abramowicz, G. Bjornsson, \& J. E. Pringle) Cambridge: Cambridge Univ. Press, 148

Schmitt, H. R. 2001, AJ, 122, 2243

Shlosman, I., Begelman, M. C., \& Frank, J. 1990, Nature, 345, 679

Shakura, N. I., \& Sunyaev, S. A. 1973, A\&A, 24, 337

Simkin, S. M., Su, H. J., \& Schwarz, M. P. 1980, ApJ, 237, 404

Toomre, A., \& Toomre, J. 1972, ApJ, 178, 623

Wada, K. 2004, in Carnegie Observatories Astrophysics Series, Vol. 1: Coevolution of Black Holes and Galaxies, (ed. L. C. Ho) Cambridge: Cambridge Univ. Press, 187 


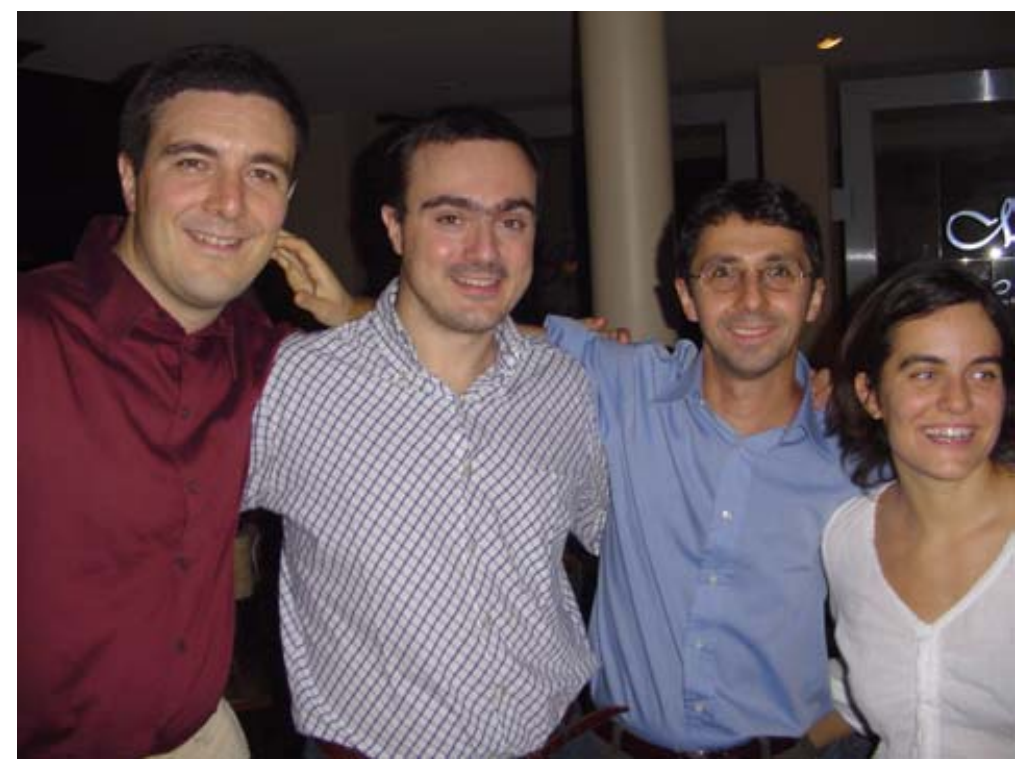

From left to right: G. Risaliti, A. Marconi, R., Maiolino and T. di Matteo.

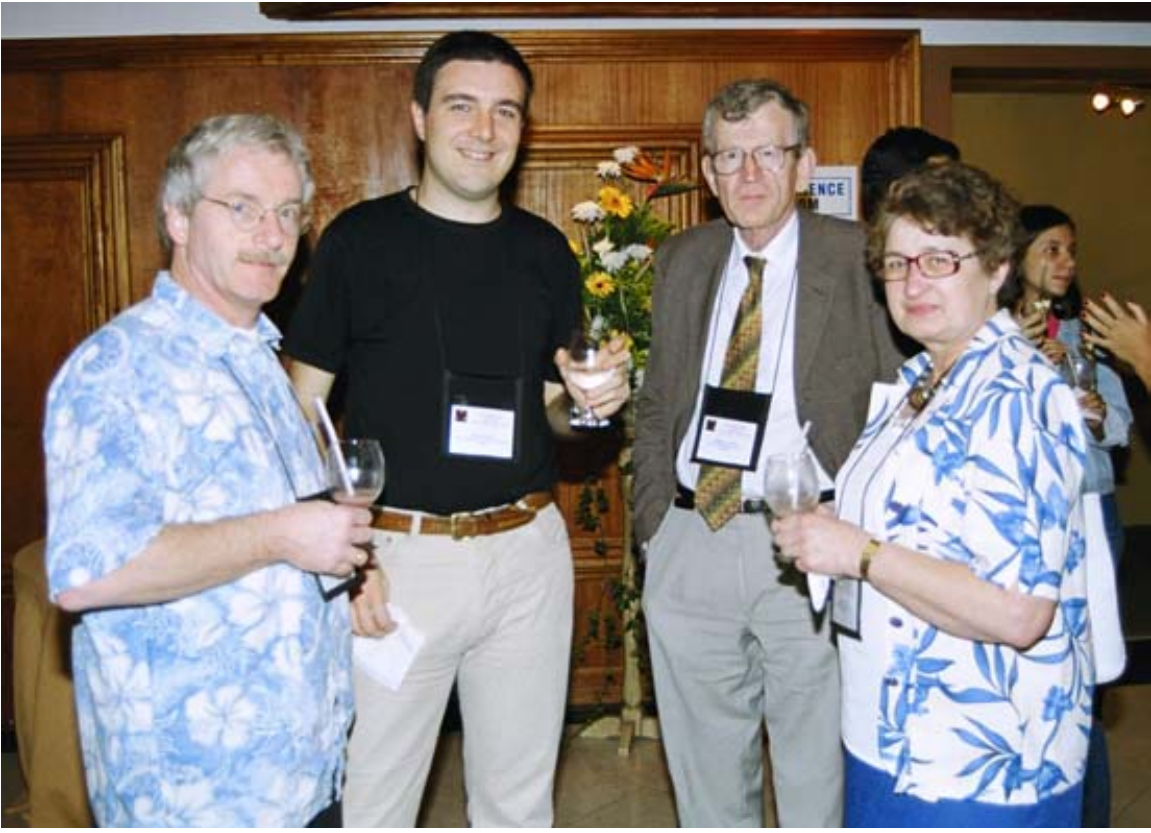

From left to right: M. Haas, G. Risaliti, P. Veron and M. Veron. 